Contents List available at VOLKSON PRESS

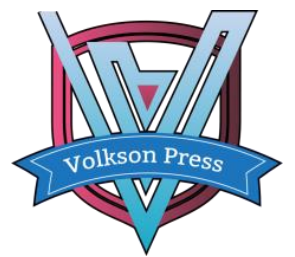

Intelligent Computing and Information Engineering (ICIE )

DOI : http://doi.org/10.26480/icie.01.2017.41.44

Journal Homepage: : https://www.intelcomp-design.com/

\title{
Approximate Growth Curve of Fetus Pancreas by Monotone Splines Regression
}

Muye Liu'1,*

\author{
Department of Statistics, University of Connecticut, 215 Glenbrook Rd., CT 06260, U.S
}

muye.liu@uconn.edu

This is an open access article distributed under the Creative Commons Attribution License, which permits unrestricted use, distribution, and reproduction in any medium, provided the original work is properly cited

\section{ARTICLE DETAILS}

\section{Article History:}

Received 12 May2017

Accepted 12 July 2017

Available online 14 September 2017

Keywords:

Pancreatic disease, growth curve,

I-splines, MLE.

\section{ABSTRACT}

This paper presents a new algorithm based on Novel Extension Rule for reasoning problems in Wumpus World. This algorithm describes these problems by propositional logic terms and solves them with Novel Extension Rule. In Wumpus World, the algorithm ascertains possible positions with pits or the wumpus in order to find a safe way to the gold. Experimental results prove its soundness and completeness.

\section{Introduction}

Epidemiological studies suggest that with the industrialization process and the continuous improvement of people's living standards, influenced by diet, smoking, drinking and other lifestyle and environmental factors, the incidence of pancreatic disease increased year by year [1]. Pancreas are secluded in the retroperitoneal, well-known far less than its neighbors stomach, duodenum, liver and gallbladder. In recent years, there is a growing number of reports about pancreatic diseases, such as acute and chronic pancreatitis, idiopathic pancreatitis, autoimmune pancreatitis, pancreatic cancer and others, and pancreatic disease gradually attracted attention.

Pancreatic endocrine tumor is a rare type of tumor, divided into functional and nonfunctioning tumor, and the main feature is the secretion of too much hormones, including insulinoma, gastrinoma, glucagon tumor, somatostatin, Vasoactive intestinal peptide and pancreatic polypeptide. A group of scientist found the expression of ghrelin and its receptors (GHSR-1a, GHSR-1b) in many pancreatic endocrine tumor tissues and did not necessarily correlate with the hormone species secreted by the tumor itself $[2,3]$.

Pancreas are rich in innervation, componented by the inner nerve and foreign nerve together, and constitute a complex neural network, controlling the external and internal secretion of the pancreas. developmental defects of Pancreatic nerve can lead to abnormal pancreatic function, and some pancreatic diseases will change the structure and distribution of pancreatic nerves, thus affecting the disease process [4].

Stathmin 1 protein is a microtubule-labile phosphoprotein that plays an important role in the regulation of microtubule motility in the cell cycle. Studies have shown that Stathmin 1 protein in many types of human malignancies were high levels of expression, such as oral cancer, esophageal cancer, gastric cancer [5]. But reports about its expression in the fetal pancreas are rare at home and abroad. Huang et al. Used immunohistochemistry and in situ hybridization to observe the expression of Stathmin 1 in fetal normal pancreas, and to provide morphological bas is for the physiological function of Stathmin $1[6]$.

In order to explore the formation and biology of the neuroendocrine system role, some researcher examined the expression of chromatin A (CgA) and neuron-specific enolase (NSE) during human pancreatic development in order to explore the formation and biology of the neuroendocrine system Role [7].

Another major research direction is children with idiopathic pancreatitis, because the cause of pancreatitis in children and adults is different. Ujjal and so on from the case group and control group of patients with peripheral blood extracted genomic DNA. The results of PCR-RFLP showed that the susceptibility gene was the main cause of chronic idiopathic and acute recurrent pancreatitis in children. The results showed that the susceptibility genes were the main genes in children with chronic idiopathic and acute recurrent pancreatitis. the reason. Acute recurrent pancreatitis is a precursor to chronic pancreatitis (CP). 1/4 of children with idiopathic acute pancreatitis (AP), especially severe, susceptible genes, should be closely followed by early detection of CP and its complications [8].

With the progress of diagnostic means, diagnosis rate of children with pancreatic disease is increasing. Congenital anatomical abnormalities, such as pancreatic dysplasia, biliary pancreatic duct confluence, ring pancreas is the main cause of chronic pancreatitis in children. Acute biliary pancreatitis is also increasing. This study through the surgical treatment of the past, this study by fitting the relationship between pancreatic parameters and fetal development time, the fetal pancreas growth curve can be used to clinically test whether the normal development of fetal pancreas [9].

\section{DATASET OF PANCREATIC PARAMETERS IN FETUS}

Our dataset contains 44 fetuses and four measurements of their pancreas, they are total length, top width, body width, tail width of pancreas, time is described by pregnant duration(days). As shown by scatter plots in Figure 1, measurements and their variance seem to have increasing trend with time. So, we use monotone splines regression to figure out their growth curve. 

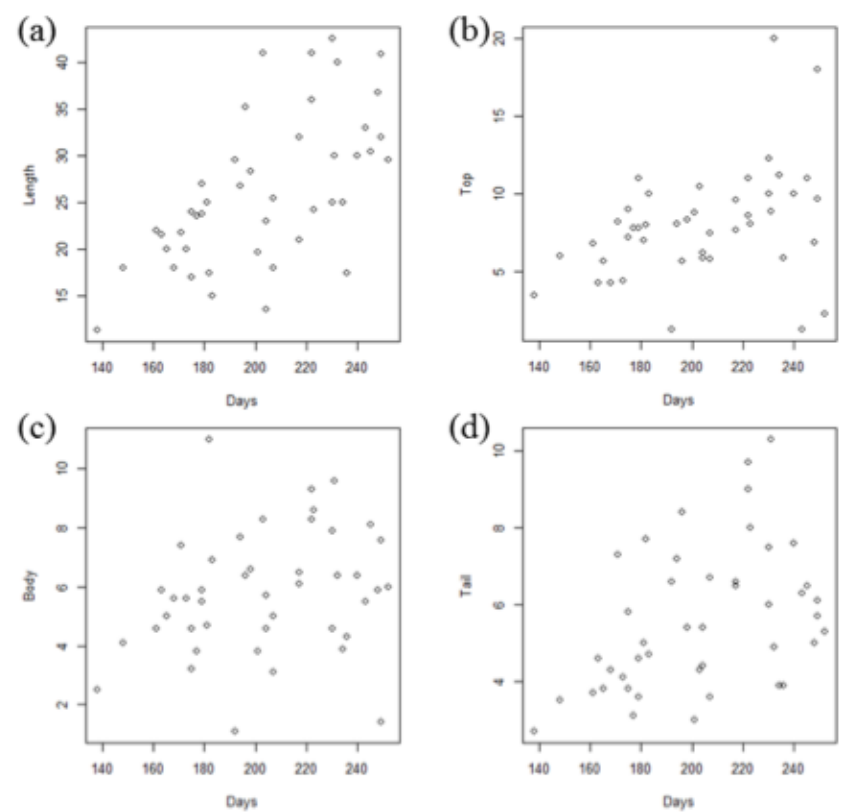

Figure 1: The relationship between pancreatic parameters in fetus and the pregnant days (a) the total length (b) the top width (c) the body width (d) the tail width of pancreas respectively

Briefly, we assume a measurement follows normal distribution with variable $\mu(t)$ and $\sigma^{2}(t)$ and then use monotone splines to represent the parameters. To estimate them, we use MLE, and use AIC as the criterion of model selection. Additionally, we study bivariate joint distribution of pairs of these four measurements.

\section{MONOTONIC SPLINES}

A very basic kinds of splines is M-splines. In Ramsay, the m order basis of M-splines is represented as following formula:

$$
\begin{aligned}
M_{i}(t \mid 1, r) & =\frac{1}{r_{i}+1-r_{i .} .} \\
M_{i}(t \mid m, r) & =\frac{m\left[\left(t-r_{i}\right) M_{i}(t \mid m-1, r)+\left(r_{i+m}-t\right) M_{i+1}(t \mid m-1, r)\right]}{(m-1)\left(r_{i+m}-r_{i}\right)} .
\end{aligned}
$$

where $r_{\tilde{I}}$ is the $i t h$ knot.

The $\mathrm{m}$ order basis of I-splines is represented by:

$I_{i}(t)=\int_{r_{i}}^{t} M_{i}(u \mid m, r) d u$

Since $M_{i}(u \mid m, r)$ is nonnegative, $I_{i}(t)$ is monotone increasing. So that for any monotone increasing piecewise polynomial or splines, it could be represented as a linear combination of I-spline basis

$f(t)=\Sigma_{i=1}^{k} a_{i} I_{i}(t)$

where $a_{i} \geq 0, I_{i}(t)$ is I-spline basis with $\mathrm{m}$ order, $\mathrm{k}$ is the degree of freedom; the spline will be smoother when $\mathrm{k}$ is increasing.

It's hard to generate these bases by our hands, but we have computer fortunately. Thanks for Wenjie Wang and his R package, we can easily generate these bases of I-splines.

\section{MAXIMUM LIKELIHOOD ESTIMATE (MLE)}

Maximal Likelihood Estimation (MLE) is a method that is still widely used. It is a statistical method based on the principle of maximum likelihood and it is in the case of the general distribution type is known. A method of estimating parameters was first proposed by German mathematician CF Gauss in 1821. The intuitive idea of the maximum likelihood principle is that there is a number of possible results in a randomized experiment. A, B, C ... If A appears in a test, it is generally assumed that the test condition is favorable to $\mathrm{A}$, ie the probability of occurrence of A is very big. Therefore, the method is to construct the log-likelihood function by using the known probability density function form, and then maximize the likelihood function to obtain the parameter estimation contained in the probability density function. Moreover, compared with other point estimates, such as least squares estimation and moment estimation, the maximum likelihood estimation fully applies the information provided by each observation value, so its estimation accuracy is extremely high, and also has an estimated consistency, asymptotic normality and asymptotic validity of many excellent statistical properties. So it is a very good parameter estimation method.

\section{APPROXIMATION}

We first assume that:

The measurement $Y_{i}$ is normal distributed with $\mu\left(t_{i}\right)$ and $\sigma^{2}\left(t_{i}\right), Y_{i}$ are independent.

$\mu\left(t_{i}\right)=\Sigma_{j=0}^{k} \beta_{j} I_{j}\left(t_{i}\right), \sigma^{2}\left(t_{i}\right)=\sum_{l=0}^{h} \theta_{l} I_{l}\left(t_{i}\right)$, 
where $\beta_{j}>0, \theta_{l}>0$ and $h, k$ are degree of freedom. Additionally, $I_{0}(t)=1$ is intercept.

We will verify normality assumption later. And under these assumptions, the log likelihood function can be represented as

$\log \left(\Pi_{i=1}^{N} f_{i}\left(y_{i}\right)\right)=C-\frac{1}{2} \sum_{i=1}^{N} \log \left(\sum_{l=0}^{h} \theta_{l} I_{l}\left(t_{i}\right)\right)-\sum_{i=1}^{N} \frac{\left(y_{i}-\sum_{j=0}^{k} \beta_{j} I_{j}\left(t_{i}\right)\right)^{2}}{2 \sum_{l=n}^{h} \theta_{l} I_{l}\left(t_{i}\right)}$.

We use MLE with constrains to estimate parameters $\beta_{j}$ and $\theta_{l}$ and then draw estimated growth curve. For different $h$ and $k$, we will get different models. Do for model selection, we choose the one with smallest AIC. The result is shown as figure 2.
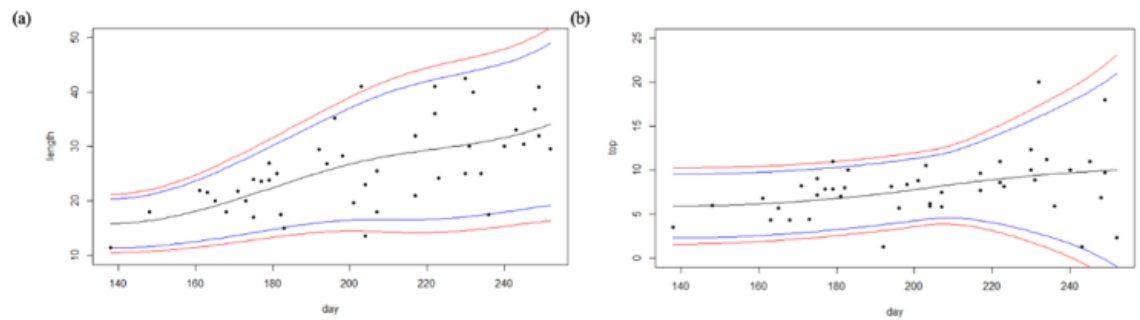

(c)

(d)
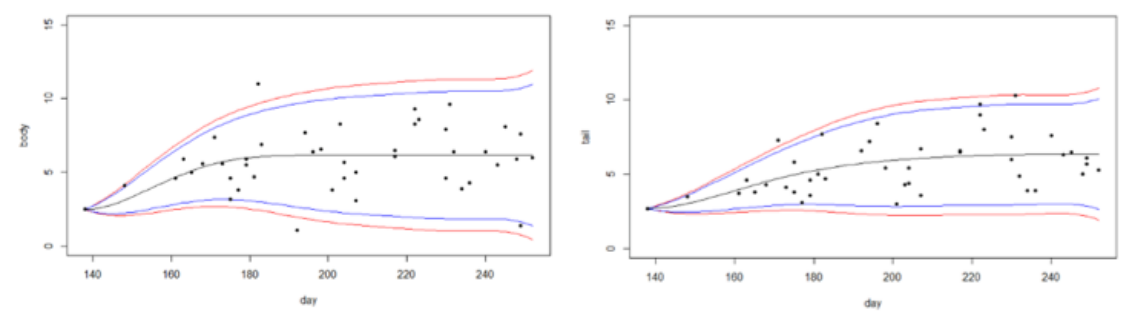

Figure 2: The estimated growth curve of different pancreatic parameters in fetus (a) the total length (b) the top width (c) the body width (d) the tail width of pancreas respectively

Two red lines represent the upper bonds and lower bonds of $95 \%$ prediction interval, two blue lines represent the upper bonds and lower bonds of $90 \%$ prediction interval. If we have observations that exceed these bonds, it may indicate some abnormity.

\section{VERIFY ASSUMPTION}

We first standardize our sample to ${ }_{w_{i}}=\frac{\underline{Y}_{i}-\mu(t),}{\sigma(t)}$, these $W_{i}$ should follow a standard normal distribution if the normality assumption is reasonable. The normal Q-Q plots are shown in figure 3 .
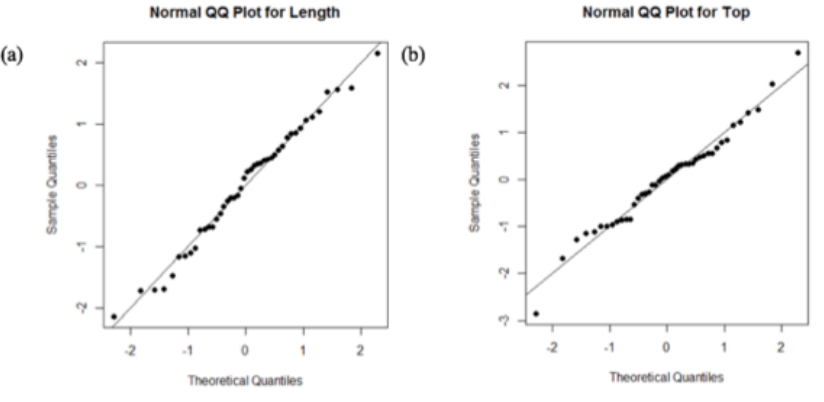

(c)
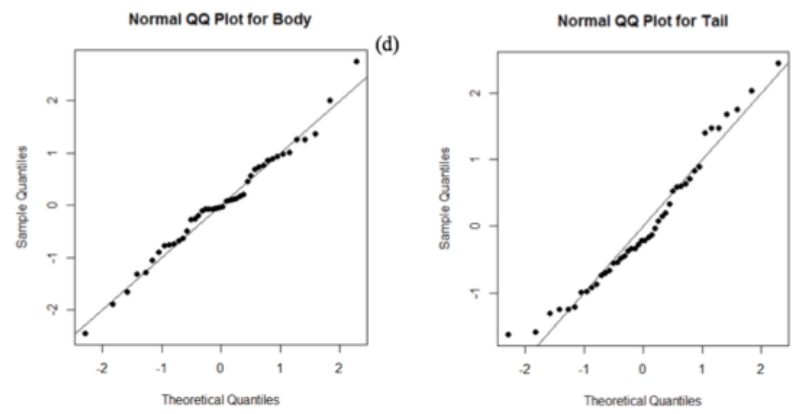

Figure 3: The result of normality assumption (a) the total length (b) the top width (c) the body width (d) the tail width of pancreas respectively From these four normal $Q-Q$ plots, we can verify that our normality assumption is reasonable.

\section{BIVARIATE DEPENDENCE}

We transfer our standard observations into pseudo-observations. We first test for independence base on $S_{n}^{\Pi}$ with $H_{0}: C \in \Pi$. We test each pair of these measurements, only between body width and tail width we can reject the null hypothesis with p-value less than 0.001 . The result of test for exchangeability shows that $S_{n}^{e x c}=0.018$ and p-value $=0.657$, and the result of test for extreme-value dependence is $S_{n}^{e v K}=-0.932$ and $p$-value $=0.351$. Common 
copulas that have these properties are t extreme value copula and Gumble copula. In practice, t-ev copula could be replaced by Gumble copula with a suitable parameter.

If we use parametric bootstrap goodness-of-fit test, we can verify if Gumble copula fit well and estimate the parameter. The p-value of the test is 0.538 so that Gumble copula fits well and the estimated fixed parameter of Gumble copula $\theta=2.974$. However, if we focus on the data under the condition that $t<180$, the estimated parameter is $\theta=1.932$. Under $180 \leq t \leq 220$, the estimated parameter is $\theta=1.542$. Under $t>220$ and fit it to Gumble copula, the estimated parameter is $\theta=2.974$. The parameter seems not fixed as we assumed. If we assume that the parameter is not fixed but depended on $t$, that is $\theta(t)=\Sigma_{j=i}^{k} \beta_{i} B_{i}(t)$. Like we did MLE previously, we can approximate a curve of $\theta(t)$ vs. $t$, which is shown in figure 4.

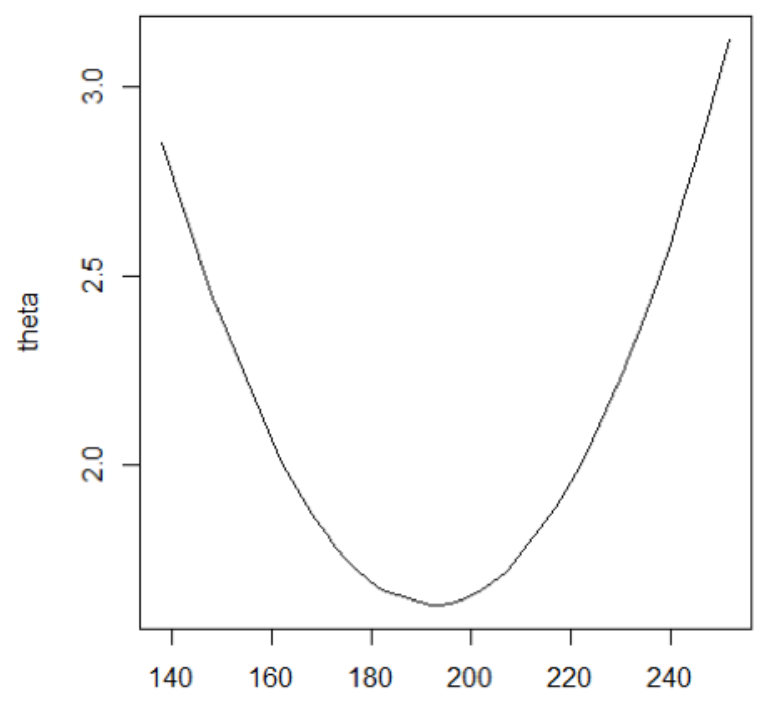

$\mathrm{t}$

\section{CONCLUSION}

Figure 4: The approximate curve of $\theta(t)$ with $t$

The dysplasia of pancreas of fetus and pancreatic hypertrophy are both disease. Particularly, dysplasia of pancreas of fetus has a very high fatality rate, so establishing a pancreas growth model has a significant sense for clinical diagnosis of such disease of pancreas. This study uses monotone splines and MLE to approximate the growth curve of measurements of fetuses' pancreas, and also give the prediction interval. The growth curve may make sense in clinical diagnosis. Additionally, we do some study about bivariate correlation and use Gumble copula to fit the copula of tail width and body width.

\section{ACKNOWLEDGEMENT}

I'm very appreciate to my tutor Jun Yan, a professor of department of statistics of University of Connecticut. He gave me many academic advices. I would like to thank Wenjie Wang and his R package splines2 also, it simplified some process in R programming.

\section{REFERENCES}

[1] Otsuki, M., Tashiro, M. 2007. Chronic pancreatitis and pancreatic cancer, lifestyle-related diseases. Internal Medicine, 46, 109-113.

[2] Volante, M., Allìa, E., Gugliotta, P., Funaro, A., Broglio, F., Deghenghi, R., Muccioli, G., Ghigo, E., Papotti, M. 2002. Expression of ghrelin and of the GH secretagogue receptor by pancreatic islet cells and related endocrine tumors. The Journal of Clinical Endocrinology and Metabolism, 87, $1300-1308$.

[3] Ekeblad, S., Lejonklou, M.H., Grimfjärd, P., Johansson, T., Eriksson, B., Grimelius, L., Stridsberg, M., Stålberg, P., Skogseid, B. 2007. Co-expression of ghrelin and its receptor in pancreatic endocrine tumours. Clinical Endocrinology, 66, 115-122.

[4] Salvioli, B., Bovara, M., Barbara, G., De, P.F., Stanghellini, V., Tonini, M., Guerrini, S., Cremon, C., Esposti, M.D., Koumandou, M., Corinaldesi, R., Sternini, C., Giorgio, R.D. 2002. Neurology and neuropathology of the pancreatic innervation. Jop Journal of the Pancreas, 3 (2), 26-33.

[5] Akhtar, J., Zhou, W., Jiang, W.P., Bi, M.M., Zhang, Z.P. 2014. Stathmin overexpression identifies high risk for lymphatic metastatic recurrence in pN0 esophageal squamous cell carcinoma patients. Journal of Gastroenterology and Hepatology, 29, 944-950.

[6] Huang, M., Zhang, X., Yu, Y., Gao, M., Jia, Y.F., Hu, W. 2015. Expression of stathmin 1 in normal fetal pancreatic tissues. Anhui Medical Journal, 36, 135137.

[7] Gao, L., Huang, Z.X., Qin, L. 2005. Expression and significance of CgA and NSE in the development of fetal pancreas. Chinese Journal of Histochemistry and Cytochemistry, 14, 309-311.

[8] Han, C.Q., Ding, Z. 2012. New research progress in pancreatic disease. Chinese Journal of Clinical Gastroenterology, 26, 379-381.

[9] Ramsay, J. 0. 1988. Monotone Regression Splines in Action. Statistical Science, 3, 425-441. 